\title{
Bone Graft Substitutes and Bone morphogenetic proteins for osteoporotic fractures: What is the Evidence?
}

E.M.M. van Lieshout (Esther) and V. Alt (Volker)

Injury: International Journal of the Care of the Injured, Volume 47 p. S43- S46.

http://dx.doi.org/10.1016/S0020-1383(16)30011-0 


\section{Bone Graft Substitutes and Bone morphogenetic proteins for osteoporotic fractures: What is the Evidence?}

Esther M.M. Van Lieshout, MSc PhD, Associate Professor ${ }^{1}$

Volker Alt, MD, PhD, Professor and Orthopaedic Trauma Surgeon ${ }^{2}$

${ }^{1}$ Trauma Research Unit Department of Surgery, Erasmus MC, University Medical Center Rotterdam, Rotterdam, The Netherlands

${ }^{2}$ Department of Trauma, Hand and Reconstructive Surgery Giessen University Hospital Giessen-Marburg, Campus Giessen, Rudolf-Buchheim-Str. 7, 35385 Giessen, Germany

\section{Corresponding author:}

Prof. Dr. med. Dr. biol. hom. Volker Alt

Department of Trauma Surgery

University Hospital Giessen-Marburg, Campus Giesen

Rudolf-Buchheim-Str. 7

35392 Giessen

Germany

email: volker.alt@chiru.med.uni-giessen.de 


\section{Abstract}

Despite improvements in implants and surgical techniques, osteoporotic fractures remain challenging to treat. Among other major risk factors, decreased expression of morphogenetic proteins has been identified for impaired fracture healing in osteoporosis. Bone grafts or bone graft substitutes are often used for stabilizing the implant and for providing a scaffold for ingrowth of new bone. Both synthetic and naturally occurring biomaterials are available. Products generally contain hydroxyapatite, tricalcium phosphate, dicalcium phosphate, calcium phosphate cement, calcium sulfate (plaster of Paris), or combinations thereof. Products have been used for the treatment of osteoporotic fractures of the proximal humerus, distal radius, vertebra, hip, and tibia plateau. Although there is generally consensus that screw augmentation increased the biomechanical properties and implant stability, the results of using these products for void filling are not unequivocal. In osteoporotic patients, BMPs have the potential impact to improve fracture healing by augmenting the impaired molecular and cellular mechanisms. However, the clinical evidence on the use of BMPs in patients with osteoporotic fractures is poor as there are no published clinical trials, case series or case studies. Even pre-clinical literature on in vitro and in vivo data is weak as most articles focus on the beneficial role for BMPs for restoration of the underlying pathophysiological factors of osteoporosis but do not look at the specific effects on osteoporotic fracture healing. Limited data on animal experiments suggest stimulation of fracture healing in ovariectomized rats by the use of BMPs. In conclusion, there is only limited data on the clinical relevance and optimal indications for the use of bone graft substitute materials and BMPs on the treatment of osteoporotic fractures despite the clinical benefits of these materials in other clinical indications. Given the general compromised outcome in osteoporotic fractures and limited alternatives for enhancement of fracture healing, clinicians and researchers should focus on this important topic and provide more data in this field in order to enable a sound clinical use of these materials in osteoporotic fractures.

Keywords: Bone graft; Bone graft substitute; Bone Morphogenetic Protein, BMP, Calcium phosphate; Calcium sulfate; Fracture; Osteoporosis; Osteoporotic fracture. 


\section{Introduction}

Despite improvements in the treatment of osteoporosis, osteoporotic fractures remain challenging to treat. Osteoporotic fractures have an impaired ability to heal $(1,2)$, and often require more time to heal (3-6). Since osteoporotic bone is less likely to heal on its own and the degree of comminution is generally high, patients often require surgery to repair the fracture. Poor bone quality, however, may complicate implant fixation. Modern angle-stable plate-screw systems and minimally invasive operative techniques have improved the stability of fixation in osteoporotic bone, but success is still not guaranteed. Due to the high porosity and low mechanical strength of osteoporotic cancellous bone, implants are often augmented with bone void fillers in order to improve outcome. Furthermore, decreased expression of bone morphogenetic proteins (BMPs) in osteoporosis combined with the essential general role of BMPs in fracture healing made BMPs attractive for improvement of impaired molecular and cellular mechanisms in osteoporotic fracture patients (7).

Bone grafts can be used to stabilize the implants and provide a scaffold for ingrowth of new bone. BMPS have the potential of de novo new bone formation due to their osteoinductive capabilities (8).

So, these materials are suitable as bone grafts fill voids, provide support, and may enhance the biological repair of the fracture or the fracture defect. This paper is aimed at providing an overview of available evidence for the use of bone graft substitutes and BMPs for the treatment of osteoporotic fractures.

\section{Bone graft substitute materials}

The limitations of autografts and allografts led to the development of bone graft substitutes. Both synthetic and naturally occurring products are available. Each has its specific composition, which determines its biological and biomechanical behavior (9-11). As such, each product will have its unique clinical indication(s).

Bone graft substitute materials provide an osteoconductive matrix, but do not contain osteogenic cells or osteoinductive growth factors. Sufficient porosity, especially the presence of interconnected pores determine the ability of bone graft materials to foster ingrowth and osteointegration. Pore sizes of at least $100 \mu \mathrm{m}$ are sufficient for osteoid formation and osseous ingrowth (12). The presence of interconnecting pores may be more critical for osseous ingrowth than the pore size per se $(13,14)$. Most bone graft substitute materials used for treating osteoporotic fractures contain calcium sulfate or calcium phosphate. 
Calcium sulfate is a self-setting, biologically inert, moldable, and osteoinductive material that provides a scaffold for osteoblasts. It rapidly dissolves (without cellular influence) in 6- 8 weeks. This may be advantageous in some cases, but if it dissolves too quickly, the augmenting effect may be lost too early, causing implant loosening.

Calcium phosphate materials include synthetic tricalcium phosphate, beta tricalcium phosphate, and coralline hydroxyapatite. The osteoconductive matrix allows osteogenic cells to create new bone under the influence of host osteoinductive factors. Calcium phosphate materials degrade at a slower rate than calcium sulfate materials, with hydroxyapatite being relatively inert. Calcium phosphate materials are available as block, granules, or cement. Blocks and granules are highly porous. They provide less initial biomechanical strength, but strength will increase upon ingrowth of new bone. Calcium phosphate cement is injected as a paste and hardens in vivo. They can be injected or molded into small bone defects and provide structural support with low porosity but good initial compressive strength.

\section{Use of bone graft substitutes for treatment of osteoporotic fractures}

Calcium sulfate and calcium phosphate cement have clear benefits when used for screw augmentation, as described in detail elsewhere (15). Clinical applications described include osteoporotic fractures of the proximal humerus, distal radius, vertebra, hip, and tibia plateau.

Both calcium sulphate and phosphate cements show promising results in the treatment of proximal humeral fractures. Minimally invasive plate fixation (internal locking system (PHILOS) augmented with calcium sulfate cement (MIIG X3; Wright Medical Technology, Arlington, TN, USA) resulted in fewer complications, less reduction loss, and better joint function than plating alone (16). MIIG 115 also resulted in fewer failed reductions when injected in the medial metaphyseal junction (17). Reduction failed in 7.1\% (1 of 14) grafted patients versus $13.3 \%$ (4 of 30) non-grafted patients. Functional outcome was good in both groups. Unfortunately, treatment allocation was not randomized. Augmentation of severely impacted valgus fractures with Norian, an injectable hydroxyapatite cement, resulted in good functional outcome (18). Augmentation was used after open reduction with screws or buttress plate fixation. All fractures united within the first year, and no patient showed loss of reductions or osteonecrosis of the humeral head.

Clinical benefit of bone graft substitute material use in osteoporotic distal humerus fractures is undecided, as studies show contradicting results. A biomechanical study showed that cement augmentation increased the biomechanical properties in volar plating. This included significant increase in cycles and load to failure, and construct stiffness at loads 
$>325 \mathrm{~N}$ as well as less fracture gap movement and screw cutting distance at the holes of the ulnar column (19). Augmentation with calcium phosphate cement also maintained fixation of unstable distal radius fractures (20). Garcés-Zarzalejo et al., on the other hand, stated that bone grafts and bone graft substitutes are not mandatory for the treatment of unstable distal radius fractures with locking compression plates (21). All 60 fractures in their study (treated without graft), healed uneventfully with no significant loss of reduction. A randomized study also showed that augmentation of metaphyseal defects with calcium phosphate bone cement after volar locking plate fixation offered no benefit over plate fixation alone (22).

Two studies showed increased screw hold in spine after augmentation $(23,24)$. Bone graft substitutes for the treatment of osteoporotic vertebral fractures have been used for kyphoplasty and vertebroplasty. Although pain and the disability scores decreased after balloon kyphoplasty with injectable calcium phosphate cement (Callos), the augmentation properties also decreased within six months, including progression of vertebral height loss and increase in kyphotic angle (25). Epidural leakage of the paste into the spinal canal was observed in 48.4\% (15 of 26) cases. Vertebroplasty using calcium phosphate cement resulted in immediate pain relief and prevented the vertebral body from late collapse and pseudoarthrosis (26). All 86 patients (99 vertebroplasties) had complete bone union within six months after surgery. Vertebroplasty using bisphosphonate-loaded calcium phosphate cement gave good results in sheep (27). Pedicle screw fixation combined with transpedicular bone grafting with demineralized bone matrix (OsteoSet, Wright Medical Technology, TN, USA) restored and maintained vertebral height successfully, and patients reported less pain at three months follow-up than pre-surgery (28).

Two studies reported that cement augmentation can increase the rotational stability and screw pull-out force in osteoporotic femoral heads $(29,30)$. Augmentation with calcium phosphate cement enhanced the fixation stability of femoral neck and trochanteric fractures (31). A meta-analysis, however, found no convincing evidence for the use of any orthobiologic bone cement in the augmentation of fractures of the hip (32).

Current evidence does not unequivocally support the need to use bone graft substitutes in the treatment of osteoporotic tibia plateau fractures. A meta-analysis showed that for tibia plateau augmentation, hydroxyapatite granules, tricalcium phosphate, demineralized bone matrix, allografts, and autografts all resulted in uneventful healing in $>90 \%$ of cases (33). The rapid degradation of calcium sulfate may be a disadvantage, as $11 \%$ of cases treated with calcium sulfate showed subsidence (34). Injectable calcium phosphate cements allow to 
support a reduced joint surface using a noninvasive procedure. Cement extrusion into a joint cavity should be prevented as these cements will not dissolve (35).

\section{Preclinical studies of the role of BMPs in osteoporosis and in osteoporotic fractures}

After the key discovery of the osteoinductive potential of BMPs to form ectopic bone reported by M. Urist in 1965 (8), more than 40 different BMPs have been described in the meantime. M. Urist himself called osteoporosis a "bone-morphogenetic auto-immune disorder" (36) and certain important interactions between BMPs in the pathomechanism of osteoporosis could be identified. Genetic polypmorphisms in BMP-2 were found to be responsible for familial osteoporosis $[37,38]$. The link between BMP-2 and osteoporosis is mainly the role of BMP-2 in the achievement of peak bone mass rather than osteolytic activity during bone loss. Both decreased anabolic activity and reduced gene expression of BMP-2 have been reported in aged rats and reduced expression of BMP-2 was confirmed in mesenchmyal stem cells obtained from confirmed in ovariectomized rats [39,40]. Pountos et al. [41] could show a positive effect of BMP-2 and BMP-7 on the osteogenic differentiation of mesenchymal stem cells obtained from patients with lower extremity fractures underscoring the hypothesis to stimulate fracture healing in these patients by application of BMPs.

Several studies were carried out to look at the therapeutical effect of BMPs to reverse bone loss in osteoporosis. Phillips et al. (2006) [42] looked at the effects of locally applied BMP-7 with different carriers into defects of ovine vertebrae bodies. BMP-7 showed a positive trend in improving mechanical strength and histomorphometric parameters of osteopenic vertebra without statistical significance, despite the absence of consistent change in BMD. Turgemann et al. (2002) [43] applied exogenous BMP-2 intraperitoneal into mice with type I and type II osteoporosis and reported an increase of trabecular bone strength combined with an increase in the number of adult mesenchymal stem cells, increase of their osteogenic activity and proliferation as well as a decrease in apoptosis. Similar results were published for the i.v. application of BMP-6 applied in aged OVX rats [44]. Significantly increased bone volume and mechanical characteristics of both the trabecular and cortical bone, the osteoblast surface, serum osteocalcin and osteoprotegerin levels, and decreased the osteoclast surface, serum Ctelopeptide, and interleukin-6 were found. Bone mineral density maintained gains for another 12 weeks after discontinuation of BMP-6 therapy.

The preclinical literature on the effects of BMPs on osteoporotic fracture healing is poor. One animal study evaluated the effects of BMP-2 in a segmental tibia defect of ovariectomized vs. sham-operated rats. The BMP-2 treated animal exhibited higher biomechanical failure loads 
and histology revealed a higher fracture healing score, including callus formation, bone union, marrow changes and cortex remodeling compared to the sham group after 8 weeks [45].

\section{Clinical evidence for the use of bone morphogenetic proteins}

Only BMP-2 and BMP-7 have been licensed for the clinical use in patients. Open tibia fractures and lumbar spinal interbody fusion are official indications for BMP-2 (InductOs ${ }^{\circledR}$, Medtronic, Tolochenaz, Switzerland; Infuse ${ }^{\circledR}$, Memphis, USA) and BMP-7 is licensed for tibial non-unions (Osigraft ${ }^{\circledR}$, Olympus Biotech; in the meantime withdrawn from the market). There are statements in the Summary Product Characteristics (SPC) both of InductOs ${ }^{\circledR}$ and of Osigraft ${ }^{\circledR}$ stating that the "The safety and efficacy of InductOs have not been demonstrated in patients with metabolic bone diseases“ and "Osigraft must not be used in patients that have a non-union resulting from pathological fractures, metabolic bone disease (or tumors)”. This limits their official use in osteoporotic fracture patients if osteoporotic fractures are defined as pathological fractures. This is mainly due to the lacking data of the use of BMPs in osteoporotic patients and not due to documented adverse effects in this entity. Despite the theoretical benefits for improvement of fracture healing in osteoporosis, there are no published clinical trials, case series or case studies of BMP-2, BMP-7 or other BMPs in patients with osteoporotic patients. Therefore, it must be stated that there is complete absence of clinical evidence for BMP application in patients with osteoporotic fractures.

\section{CONCLUSION}

Given the generally compromised outcome in osteoporotic fractures and limited alternatives for enhancement of fracture healing, it should be assumed that bone graft substitute materials BMPs have been extensively studied for this entity. Therefore, it is more than disappointing that there is only very limited clinical data available on this indication that do not allow for an evidence-based algorithm. With a growing elderly population and limited treatment alternatives, the tremendous challenge of treating patients with osteoporotic fractures will become increasingly important and both bone graft materials and BMPs are still a viable option. Researchers and clinicians should grasp the opportunity to contribute towards this important topic and seriously evaluate the potential benefits and harms of these materials in osteoporotic fractures. 


\section{REFERENCES}

1. Thormann U, El Khawassna T, Ray S, Duerselen L, Kampschulte M, Lips K, et al. Differences of bone healing in metaphyseal defect fractures between osteoporotic and physiological bone in rats. Injury 2014;45(3): 487-93.

2. Lips KS, Kauschke V, Hartmann S, Thormann U, Ray S, Kampschulte M, et al. Podoplanin immunopositive lymphatic vessels at the implant interface in a rat model of osteoporotic fractures. PLoS One 2014;8(10): e77259.

3. Giannoudis P, Tzioupis C, Almalki T, Buckley R. Fracture healing in osteoporotic fractures: is it really different? A basic science perspective. Injury 2007;38 Suppl 1: S90-9.

4. Egermann M, Schneider E, Evans CH, Baltzer AW. The potential of gene therapy for fracture healing in osteoporosis. Osteoporos Int 2005;16 Suppl 2: S120-8.

5. Namkung-Matthai H, Appleyard R, Jansen J, Hao Lin J, Maastricht S, Swain M, et al. Osteoporosis influences the early period of fracture healing in a rat osteoporotic model. Bone 2001;28(1): 80-6.

6. Lill CA, Hesseln J, Schlegel U, Eckhardt C, Goldhahn J, Schneider E. Biomechanical evaluation of healing in a non-critical defect in a large animal model of osteoporosis. $\mathrm{J}$ Orthop Res 2003;21(5): 836-42.

7. Kanakaris NK, Petsatodis G, Tagil M, Giannoudis PV. Is there a role for bone morphogenetic proteins in osteoporotic fractures? Injury. 2009;40 Suppl 3: S21-6.

8. $\quad$ Urist MR. Bone: formation by autoinduction. Science 1965;150:893-9.

9. Van der Stok J, Van Lieshout EM, El-Massoudi Y, Van Kralingen GH, Patka P. Bone substitutes in the Netherlands - a systematic literature review. Acta Biomater 2011;7(2): 739-50.

10. Watson JT, Nicolaou DA. Orthobiologics in the augmentation of osteoporotic fractures. Curr Osteoporos Rep 2015;13(1): 22-9.

11. Sterling JA, Guelcher SA. Biomaterial scaffolds for treating osteoporotic bone. Curr Osteoporos Rep 2014;12(1): 48-54.

12. Kuhne JH, Bartl R, Frisch B, Hammer C, Jansson V, Zimmer M. Bone formation in coralline hydroxyapatite. Effects of pore size studied in rabbits. Acta Orthop Scand 1994;65(3): 246-52. 
13. De Long WG, Jr., Einhorn TA, Koval K, McKee M, Smith W, Sanders R, et al. Bone grafts and bone graft substitutes in orthopaedic trauma surgery. A critical analysis. $\mathrm{J}$ Bone Joint Surg Am 2007;89(3): 649-58.

14. Nakahara H, Goldberg VM, Caplan AI. Culture-expanded periosteal-derived cells exhibit osteochondrogenic potential in porous calcium phosphate ceramics in vivo. Clin Orthop Relat Res 1992(276): 291-8.

15. Larsson S, Procter P. Optimising implant anchorage (augmentation) during fixation of osteoporotic fractures: is there a role for bone-graft substitutes? Injury 2011;42 Suppl 2: S72-6.

16. Liu ZZ, Zhang GM, Ge T. Use of a proximal humeral internal locking system enhanced by injectable graft for minimally invasive treatment of osteoporotic proximal humeral fractures in elderly patients. Orthop Surg 2011;3(4): 253-8.

17. Lee CW, Shin SJ. Prognostic factors for unstable proximal humeral fractures treated with locking-plate fixation. J Shoulder Elbow Surg 2009;18(1): 83-8.

18. Robinson CM, Page RS. Severely impacted valgus proximal humeral fractures. Results of operative treatment. J Bone Joint Surg Am 2003;85-A(9): 1647-55.

19. Hogel F, Mair S, Eberle S, Weninger P, von Oldenburg G, Augat P. Distal radius fracture fixation with volar locking plates and additional bone augmentation in osteoporotic bone: a biomechanical study in a cadaveric model. Arch Orthop Trauma Surg 2013;133(1): 51-7.

20. Zimmermann R, Gabl M, Lutz M, Angermann P, Gschwentner M, Pechlaner S. Injectable calcium phosphate bone cement Norian SRS for the treatment of intraarticular compression fractures of the distal radius in osteoporotic women. Arch Orthop Trauma Surg 2003;123(1): 22-7.

21. Garces-Zarzalejo C, Sanchez-Crespo MR, Penas-Diaz F, Ayala-Gutierrez H, Sanz Gimenez-Rico JR, Alfonso-Fernandez A, et al. Distal radius fractures: Should we use supplemental bone grafts or substitutites in cases of severe osteoporotic or conminution? Rev Esp Cir Ortop Traumatol 2014.

22. Kim JK, Koh YD, Kook SH. Effect of calcium phosphate bone cement augmentation on volar plate fixation of unstable distal radial fractures in the elderly. J Bone Joint Surg Am 2011;93(7): 609-14.

23. Bullmann V, Liljenqvist UR, Rodl R, Schulte TL. [Pedicle screw augmentation from a biomechanical perspective]. Orthopade 2010;39(7): 673-8. 
24. Chen LH, Tai CL, Lai PL, Lee DM, Tsai TT, Fu TS, et al. Pullout strength for cannulated pedicle screws with bone cement augmentation in severely osteoporotic bone: influences of radial hole and pilot hole tapping. Clin Biomech (Bristol, Avon) 2009;24(8): 613-8.

25. Ryu KS, Shim JH, Heo HY, Park CK. Therapeutic efficacy of injectable calcium phosphate cement in osteoporotic vertebral compression fractures: prospective nonrandomized controlled study at 6-month follow-up. World Neurosurg 2010;73(4): 408-11.

26. Nakano M, Hirano N, Zukawa M, Suzuki K, Hirose J, Kimura T, et al. Vertebroplasty Using Calcium Phosphate Cement for Osteoporotic Vertebral Fractures: Study of Outcomes at a Minimum Follow-up of Two Years. Asian Spine J 2012;6(1): 34-42.

27. Verron E, Pissonnier ML, Lesoeur J, Schnitzler V, Fellah BH, Pascal-Moussellard H, et al. Vertebroplasty using bisphosphonate-loaded calcium phosphate cement in a standardized vertebral body bone defect in an osteoporotic sheep model. Acta Biomater 2014;10(11): 4887-95.

28. Li D, Huang Y, Yang $H$, Sun $T$, Wu Y, Li X, et al. Short-segment pedicle instrumentation with transpedicular bone grafting for nonunion of osteoporotic vertebral fractures involving the posterior edge. Eur J Orthop Surg Traumatol 2013;23(1): 21-6.

29. Erhart S, Schmoelz W, Blauth M, Lenich A. Biomechanical effect of bone cement augmentation on rotational stability and pull-out strength of the Proximal Femur Nail Antirotation. Injury 2011;42(11): 1322-7.

30. Augat P, Rapp S, Claes L. A modified hip screw incorporating injected cement for the fixation of osteoporotic trochanteric fractures. J Orthop Trauma 2002;16(5): 311-6.

31. Moroni A, Larsson S, Hoang Kim A, Gelsomini L, Giannoudis PV. Can we improve fixation and outcomes? Use of bone substitutes. J Orthop Trauma 2009;23(6): 422-5.

32. Lindner T, Kanakaris NK, Marx B, Cockbain A, Kontakis G, Giannoudis PV. Fractures of the hip and osteoporosis: the role of bone substitutes. J Bone Joint Surg $\mathrm{Br}$ 2009;91(3): 294-303.

33. Goff T, Kanakaris NK, Giannoudis PV. Use of bone graft substitutes in the management of tibial plateau fractures. Injury 2013;44 Suppl 1: S86-94.

34. Bajammal SS, Zlowodzki M, Lelwica A, Tornetta P, 3rd, Einhorn TA, Buckley R, et al. The use of calcium phosphate bone cement in fracture treatment. A meta-analysis of randomized trials. J Bone Joint Surg Am 2008;90(6): 1186-96. 
35. Mauffrey C, Fader R, Hammerberg EM, Hak DJ, Stahel PF. Incidence and pattern of technical complications in balloon-guided osteoplasty for depressed tibial plateau fractures: a pilot study in 20 consecutive patients. Patient Saf Surg 2013;7(1): 8.

36. Urist MR, Hudak RT, Huo YK, Rasmussen JK. Osteoporosis: a bone morphogenetic protein auto-immune disorder. Prog Clin Biol Res 1985;187:77-96.

37. Reneland RH1, Mah S, Kammerer S, Hoyal CR et al., Association between a variation in the phosphodiesterase 4D gene and bone mineral density. BMC Med Genet. 2005;6:9.

38. Styrkarsdottir U, Cazier JB, Kong A, et al. Linkage of osteoporosis to chromosome 20p12 and association to BMP2. PLoS Biol 2003;1:E69

39. Fleet JC, Cashman K, Cox K, Rosen V. The effects of aging on the bone inductive activity of recombinant human bone morphogenetic protein-2. Endocrinology 1996;137:4605-10.

40. Zhou S, Zilberman Y, Wassermann K, et al. Estrogen modulates estrogen receptor alpha and beta expression, osteogenic activity, and apoptosis in mesenchymal stem cells (MSCs) of osteoporotic mice. J Cell Biochem Suppl 2001;Suppl 36: 144-55.

41. Pountos I, Georgouli T, Henshaw K, Bird H, Jones E, Giannoudis PV. The effect of bone morphogenetic protein-2, bone morphogenetic protein-7, parathyroid hormone, and platelet-derived growth factor on the proliferation and osteogenic differentiation of mesenchymal stem cells derived from osteoporotic bone. J Orthop Trauma. 2010; 24:552-6.

42. Phillips FM, Turner AS, Seim 3rd HB, et al. In vivo BMP-7 (OP-1) enhancement of osteoporotic vertebral bodies in an ovine model. Spine J 2006;6:500-6.

43. Turgeman G, Zilberman Y, Zhou S, et al. Systemically administered rhBMP-2 promotes MSC activity and reverses bone and cartilage loss in osteopenic mice. J Cell Biochem 2002;86:461-74.

44. Simic P, Culej JB, Orlic I, et al. Systemically administered bone morphogenetic protein6 restores bone in aged ovariectomized rats by increasing bone formation and suppressing bone resorption. J Biol Chem 2006;281:25509-21.

45. Sarban S, Senkoylu A, Isikan UE, Korkusuz P, Korkusuz F. Can rhBMP-2 containing collagen sponges enhance bone repair in ovariectomized rats?: a preliminary study. Clin Orthop Relat Res. 2009 Dec;467:3113-20. 